\title{
Une communication globale dans un environnement morcele, l'interpretation des signes
}

Michèle Gabay et Sophie Pène

\section{(2) OpenEdition}

\section{Journals}

Édition électronique

URL : http://journals.openedition.org/communicationorganisation/1587

DOI : 10.4000/communicationorganisation. 1587

ISSN : 1775-3546

Éditeur

Presses universitaires de Bordeaux

\section{Édition imprimée}

Date de publication : 1 novembre 1992

ISSN : 1168-5549

Référence électronique

Michèle Gabay et Sophie Pène, " Une communication globale dans un environnement morcele, l'interpretation des signes », Communication et organisation [En ligne], 2 | 1992, mis en ligne le 26 mars 2012, consulté le 19 avril 2019. URL : http://journals.openedition.org/

communicationorganisation/1587 ; DOI : 10.4000/communicationorganisation.1587

Ce document a été généré automatiquement le 19 avril 2019

(c) Presses universitaires de Bordeaux 


\title{
Une communication globale dans un environnement morcele, l'interpretation des signes
}

\author{
Michèle Gabay et Sophie Pène
}

1 Ce travail est le résultat d'une approche théorique à la frontière entre la communication en entreprise et une analyse organisationnelle ; nous illustrons notre réflexion par l'étude du cas d'un groupe d'hôteliers et de restaurateurs indépendants: Best Western France. Nous nous proposons de montrer que l'évolution d'une communication interne vers la communication globale doit être centrée autour d'un Plan Commun d'Action ou P.C.A.

2 La méthodologie d'élaboration de ce P.C.A serait la suivante : à partir d'une recherche sur les interactions entre la structure de l'entreprise, les styles de management des dirigeants et les places des individus, telles qu'elles sont données ou imposées dans l'espace de travail ou dans la relation écrite, un conseil en communication est à même de construire avec le directoire un P.C.A qui mobilise les différents acteurs autour d'un dessein de communication globale. Notre hypothèse fait du P.C.A la condition même de la communication globale. Il est construit avec le directoire grâce aux indications données par les acteurs, forces vives de l'entreprise, au cours de l'approche de terrain.

3 Cette étude a été menée pendant une durée d'un an et demi. Nous avons constitué un observatoire au siège et procédé à des entretiens individuels de tous les chefs de service et de la direction, des entretiens par groupe de quinze, de tous les hôteliers, à une analyse des structures administratives et organisationnelles. Nous avons exploré les productions écrites du groupe sur deux ans tant en communication externe qu'en communication interne. De plus, nous avons étudié l'image du groupe à travers les écrits publiés dans les 
guides: Michelin, Gault et Millau, le Bottin Gourmand, le Routard et les guides professionnels de tourisme.

4 Enfin, nous avons dépouillé et analysé les commentaires consignés dans des questionnaires remplis par 850 clients de Best Western France. Notre objectif était double :

- une mission de conseil, qui portait sur l'élaboration d'une campagne de notoriété du groupe, nous avons fait un diagnostic de la situation et avons proposé une recherche sur les interactions entre la structure de l'entreprise, les styles de management des dirigeants et la communication globale.

- une finalité de recherche et de réflexion sur le P.C.A qui aboutit à définir les conditions de sa mise en place.

\section{Le plan commun d'action}

$5 \quad$ Nous ne retenons pas le concept de projet d'entreprise, trop utilisé dans des acceptions différentes, et taxé de valeurs connotatives démagogiques.

6 Pour nous le P.C.A est un plan qui s'établit à long et moyen terme. Il est commun dans le sens où il est élaboré avec les acteurs de l'entreprise, selon une politique dépendante de l'environnement économique et social de l'entreprise. Il propose des actions à développer pour réaliser la planification annoncée. Certaines conditions doivent être remplies pour qu'il y ait véritablement P.C.A.

7 Selon nous il doit exister :

- une dimension prospective et projective de l'entreprise en congruence avec son objet matérialisée par un projet, un but, un objectif à atteindre ;

- une convergence dans la volonté partagée de vaincre les obstacles (concurrence, modification des goûts et habitudes des clients, crise, produits éphémères...) pour atteindre ce but ;

- un système de valeurs identifié et partagé par les acteurs de l'entreprise et identifiables. Ces valeurs définies et représentatives d'une adhésion des acteurs seront repérables par des signes, indicateurs réels ou symboliques. La cohérence de ces signes est la production d'une signification externe, la cohésion fait sens interne ;

- une planification des axes de réalisation. Cette planification détermine des priorités et se fixe un programme chronologique d'action.

La communication globale est la mise en place d'une stratégie de communication, à la fois, interne et externe, qui sera en symbiose avec le P.C.A.

La fonction première d'un responsable de communication est de rechercher à faciliter l'élaboration d'un P.C.A; il peut jouer le rôle d'accoucheur, de médiateur, dans la conception. Si le P.C.A n'existe pas, mais que des objectifs et des valeurs non explicites traversent l'institution, le responsable de communication aide à l'extériorisation des signes qui favorisent l'identification de ces valeurs. Son rôle, outre l'information sur ces signes, s'oriente vers une dynamisation des échanges d'informations, pour instaurer une communication interactive. C'est aussi de diffuser et de promouvoir les signes qui seront l'expression du P.C.A.

10 Les destinataires sont les acteurs de l'entreprise ; ils représentent les meilleurs vecteurs des valeurs de l'entreprise et le public extérieur. 
hôteliers, quelques anciens, quittent le groupe, des nouveaux rejoignent Best Western
France. De plus en plus, le groupe compte des financiers, investisseurs dont les hôtels
sont gérés par des directeurs : l'identité évolue.

\section{Qu'en est il aujourd'hui ?}

1992 Best Western France compte 160 hôtels et restaurants repartis sur toute la France dont 40 à Paris et dans la région parisienne.

Best Western France fait partie du groupement Best Western International : 3500 hôtels répartis dans 62 pays dont 1500 hôtels aux Etats Unis.

Best Western France est une société anonyme coopérative à capital variable qui regroupe des hôteliers indépendants en une structure dont les buts sont:

- faire bénéficier les adhérents des flux de clientèle induits par le système de réservation national et international ;

- mettre en commun les ressources du groupe pour offrir une synergie commerciale de démarchage de clientèle, pour bénéficier d'une politique de communication ;

- utiliser les services communs d'une centrale d'achats.

\section{Structures de Best Western France}

Best Western France se présente comme une communauté d'adhérents, les hôteliers, placée sous le pouvoir de l'administration centrale de Best Western siège. 

l'architecture, à leur emplacement sur le territoire : villes d'affaires, villes touristiques, campagne, mer, montagne, Paris ou province, à leur capacité d'accueil: de 15 à 200 chambres. Ils s'adressent à une clientèle française et étrangère, d'affaire ou de tourisme familial.

\section{Eléments morcelés d'une organisation éclatée. Structure schizophrénique.}

Si nous prenons la symbolique du corps pour représenter une organisation et considérons qu'elle est constituée d'éléments : les membres ou acteurs de l'entreprise, qui vivent dans un environnement de signes matériels ou symboliques, et qui constituent le corps de l'entreprise, quelle approche pouvons nous proposer?

37 La perception du corps chez le nourrisson, jusqu'à 6 ou 7 mois, est morcelée, il perçoit chaque partie de son corps : ses mains, ses pieds comme des objets étrangers qui lui sont extérieurs. Ce n'est que plus tard qu'il construit son schéma corporel, image tridimensionnelle que chacun a de soi-même, et prend conscience de son corps comme 
unifié. Lacan pense que le nourrisson a une vision dramatique de la perception de son corps, elle est morcelée et lui fait vivre des manifestations fantasmatiques et insolites. Il vit « un corps morcelé, éclaté, il est en proie à des phénomènes d'éviration, de mutilation, de démembrement, de dislocation, d'éventrement, de dévoration $»^{1}$. Nous développons les différentes théories dans "Magnétoscope et formation à l'observation des attitudes corporelles, contribution à une étude du langage du corps $»^{2}$

L'adolescence est également un moment charnière dans la construction de l'image de soi, en effet le corps se transforme, les membres s'allongent, la voix change, l'ex-enfant lorsqu'il se voit dans un miroir, ne se reconnaît plus. L'adolescent est soumis à des pulsions internes; la puberté provoque de profonds changements anatomiques et physiologiques mais aussi psychiques. Ces modifications brutales de son corps paraissent inquiétantes parce qu'inexpliquées et incontrôlables par lui. Si nous nous référons à l'historique de Best Western France : le groupe vit actuellement une crise de croissance et recherche des structures matures. Les hôteliers ne contrôlent plus l'organisation qui grandit. Certains malades mentaux n'ont plus la perception, la sensation de leur moi corporel unifié, leur corps leur est étranger. Une situation éclatée est porteuse d'un univers schizophrénique anxiogène. Si nous transposons cette analyse à celle d'une institution, nous pouvons penser que la représentation symbolique d'un corps unifié ne peut se faire que lorsqu'il y a ancrage avec des signes repérables et identifiables. Le P.C.A contribuera à créer cette image unifiée; sa pertinence dépend de la prise en compte des structures de l'entreprise, des attitudes des membres de la direction, à la tête de l'entreprise, et de la production de signes d'identification développée par la communication globale. "Signes" peut être pris non seulement dans le sens d'indices matériels, ou symboliques, mais aussi nous pouvons retenir la succession d'événements réels ou imaginaires qui s'inscrivent dans un réel organique pour devenir signe.

L'exemple de Best Western France montre que des signes de dissociation ne contreviennent pas à un P.C.A, à condition que leur valeur soit mise au jour. Il s'agit alors d'évaluer :

- les relations au sein de l'équipe de direction et les interactions avec le responsable de la communication

- les adhérents hôteliers

- la structuration de l'espace

- la communication écrite interne et externe.

\section{Les relations au sein de l'équipe de direction de Best Western France}

40 L'équipe de direction est composée du président, du directeur, du conseil d'administration à laquelle nous ajoutons le chef du service de la communication car le président souhaite qu'elle soit « son bras gauche».

Pour analyser la structure et les interactions de l'équipe de direction nous avons retenu 3 variables qui sont:

- Les objectifs affichés pour le groupe,

- les enjeux personnels,

- le style de management, avec une dimension personnelle. 

regroupement d'hôteliers. Il est quasi bénévole. Ses objectifs pour le groupe sont de doubler en 3 ans le nombre d'adhérents, de définir des critères de qualité des hôtels à recruter et d'améliorer la qualité de ceux qui sont déjà adhérents de Best Western France. Il affiche des valeurs de groupe. Il est très connu et reconnu dans le milieu hôtelier, il est d'ailleurs, professionnel de l'hôtellerie, lui-même propriétaire et actionnaire de deux hôtels.

C'est un stratège, homme de compromis, il prend des décisions après de longues réflexions. Les hôteliers le respectent et lui font confiance, ils lui accordent du charisme. C'est un leader apaisant (3) selon la classification de H. Montagner. Il privilégie la discussion conviviale pour régler les litiges. Il a le souci d'écouter et de prendre en considération les remarques des adhérents et du personnel. Son style de management est tourné vers la volonté d'obtenir un consensus, d'éviter les ruptures, il privilégie la négociation à l'agir. Il « laisse le temps au temps ». Ses enjeux personnels se fondent avec les objectifs du groupe : le groupe est «son enfant ", il l'a porté au cours de son histoire. Son souci : laisser le groupe intègre à son successeur.

Il a assumé avec le Conseil d'administration la responsabilité d'anciens recrutements d'hôteliers pour lesquels les critères de sélection étaient plus subjectifs qu'objectifs et rationnels.

Le directeur actuel, ancien directeur commercial dans une chaîne d'hôtels est recruté en 1989 à la suite d'un conflit, entre ce président et quelques hôteliers, qui s'était entre autres choses, soldé par la révocation de l'ancien directeur.

Ses objectifs explicites pour le groupe sont identiques à ceux exprimés par le président mais lors de son recrutement il se fixe un premier objectif : liquider le contentieux des créances de Best Western France qui étaient les cessations de paiement de cotisation des adhérents hôteliers. Western avec les autres pays.

8 Certains de ses enjeux personnels favorisent le développement du groupe comme par exemple ceux de devenir un chef d'entreprise dont la dimension permette des influences directes et des changements rapides dans un environnement connu, de transférer des méthodes acquises dans une chaîne à un groupe d'hôteliers indépendants où le produit est porteur mais dont l'activité commerciale est faible.

Outre les objectifs de développement de l'entreprise, le directeur vise des enjeux personnels. Il souhaite être leader reconnu, il manifeste l'envie de gagner, la passion pour le monde de l'hôtellerie, la volonté de régner de façon inconditionnelle - il a licencié tout le personnel de l'ancienne équipe - sur une équipe dont il a recruté tous les membres.

Il consent d'ailleurs une baisse de revenu pour assumer les fonctions de directeur du groupe dans une situation de crise.

51 Son style de management est l'action, c'est un leader agressif selon H. Montagner ${ }^{3}$, il prend très rapidement et sans concertation des décisions qu'il met à exécution immédiatement. Il assume avec détermination et autorité ses actes, même s'ils sont durs pour les adhérents, par exemple l'exclusion d'un hôtelier. Il mène avec ardeur son personnel : « ou ça passe ou ça casse », « si vous n'êtes pas content c'est la porte » ou bien « c'est ça ou je vous vide ».

Communication et organisation, 2 | 1992 
52 Le directeur est absorbé par le quotidien; c'est un tacticien qui n'a ni le temps ni les outils de gestion qui permettent d'élaborer des stratégies à long terme. Sa conduite de direction est saccadée et provoque des changements par crise puis ruptures (cf le taux élevé de rotation du personnel). Pour lui cet « indicateur ne veut rien dire, c'est celui que l'on a dans l'hôtellerie ».

Ses objectifs actuels annoncés : réduire les dépenses et contraindre les acteurs à adhérer à ses valeurs de la gestion dans l'hôtellerie.

La responsable de la communication est embauchée depuis 6 mois (au début de la recherche). Elle a été recrutée à la fois par le président et le directeur, c'est la seule fonction pour laquelle le président souhaite participer au choix du candidat qui occupera le poste.

Elle a une connaissance du milieu touristique et des journalistes du tourisme, son objectif premier est de créer, ou d'améliorer quand ils existent, les supports de communication externe. Elle souhaite développer la communication interne entre Best Western Siège et les hôteliers, et faciliter la communication interne au siège.

57 Son enjeu est de se prouver qu'elle peut assumer cette lourde fonction dans un environnement difficile avec des moyens limités. Elle souhaite être reconnue dans le milieu de la communication externe et de l'édition touristique de façon affirmée; par exemple, elle vise un prix de la meilleure publication.

58 Ses fonctions ne sont pas clairement définies, son territoire de communication pas bien délimité ; par exemple pour l'organisation du congrès annuel Best Western France, rencontre annuelle des adhérents avec tenue de l'assemblée générale, elle n'est pas consultée ni pour la conception ni pour la mise en place de la logistique de communication.

Elle n'est pas informée de la conception de produits de promotion nouveaux.

60 Son mode d'expression est direct et spontané, ce qui n'exclut pas quelquefois une apparente agressivité. Son style de management répond à des impulsions affectives, elle recherche l'adhésion mais n'a pas recours au compromis. Elle a besoin d'être estimée et d'avoir des repères pour se sentir en sécurité pour travailler.

61 Ses enjeux dans l'entreprise ne vont pas vers une perspective de carrière, aussi est-elle indépendante par rapport au directeur et au président.

Elle n'affiche pas un style d'apparence normée par exemple dans sa façon de s'habiller, de travailler.

Elle semble perçue comme un danger de changement par l'assistante du directeur. Vue de l'extérieur, elle est atypique, en outre certains hôteliers connaissent mal son rôle. La responsable de communication semble totaliser les caractéristiques des deux personnalités celle du directeur et celle du président, et focaliser les projections fantasmatiques de leurs différences.

64 La direction est bicéphale mais les deux têtes ne vont pas dans le même sens. Lorsqu'il y a des conflits le président et le directeur n'ont pas les mêmes approches; les décisions du directeur tombent " comme un couperet » quant à celles du président " elles traînent ".

Les conflits entre les têtes sont larvés, pas exprimés de façon explicite, sinon rapportés par les autres acteurs. La responsable de communication est associée aux décisions de façon conflictuelle et avec des ruptures temporelles. Elle est soumise à des injonctions 
paradoxales Watlzawick, Helmick Beavin et Don. D. Jackson (1979) ${ }^{4}$ d'autonomie qui la déstabilisent : " vous êtes totalement responsable de votre service » mais par ailleurs : le directeur dit «je veux voir tout le courrier, le signer». Dans le combat des chefs la responsable de communication joue à son insu le rôle du bouc émissaire.

Face aux options divergentes des chefs, la responsable de la communication a eu besoin d'une médiation, elle a introduit l'équipe de conseils et de chercheurs en communication dans l'entreprise après accord du directeur, puis du président.

Les stratégies ne sont pas confrontées puis discutées pour être acceptées et les moyens pour leur mise en œuvre ne peuvent pas dans ces conditions être harmonisés.

La responsable de la communication, non reconnue, ne peut pas jouer le rôle d'accoucheur, ou même de relais dans la mise en place d'un plan de communication dans une structure éclatée et conflictuelle.

Les différences dans le style de management, ou la non référence aux mêmes valeurs ne condamnent pas une institution à l'asphyxie lorsqu'elles sont acceptées comme complémentaires. La complémentarité devient au contraire le levier d'une synergie productrice de forces de changement. Les différences représentent alors le ferment d'un champ propice à l'élaboration d'une stratégie, d'un P.C.A.

La complémentarité doit être reconnue comme un enrichissement de la palette des actions de management et de communication.

1 De plus si à la tête, et avec les membres, il n'existe pas de cohésion interne vers la reconnaissance des différences comme complémentaires, alors les signes offerts à voir à l'extérieur marquent un manque de cohérence. Cet affichage, nous le verrons plus loin, se manifeste par des signes extérieurs porteurs de valeurs discordantes. Il est alors impossible, pour des agents extérieurs, par exemple ici les hôteliers par rapport au siège, puis les clients face à la politique de communication externe, de percevoir des messages contenant des valeurs crédibles de forces d'unification.

\section{Comment l'aspect morcelé est-il accru par l'environnement hôtelier des adhérents ?}

Le fonds des premiers adhérents a vingt ans et est resté attaché à la première marque : MAPOTEL (l'enseigne MAPOTEL existe encore sur la façade de certains hôtels) représentative à leur yeux d'une haute qualité de l'hôtellerie française. Rejoints par des entrepreneurs indépendants, créateurs de leur hôtel, gérant des établissement familiaux de tradition ou investisseurs, ils affrontent ensemble une double contrainte :

73 - la nécessité de se développer pour maîtriser les coûts de fonctionnement du groupe et faire face à la concurrence des chaînes intégrées. Celles-ci bénéficient d'un capital de notoriété récent mais en augmentation rapide et d'une stratégie commerciale mûrie depuis une dizaine d'années.

74 - la volonté de préserver l'originalité d'établissements de tradition, le rejet de toute intrusion dans la gestion de leur entreprise, le refus des marques d'appartenance au groupe qui semblent compromettre toute tentative concertée de développement. 


\section{Souhaits d'indépendance}

En outre ces hôteliers souhaitent rester «indépendants », terme qui intervient dans la définition de l'objet Best Western France : « groupe d'hôtels indépendants », ceci explique qu'ils n'aient pas choisi des chaînes d'hôtellerie intégrées. Pour chaque hôtel, un souhait commun : conserver sa spécificité, son apparence externe, son identité. Nous pouvons y voir un choix stratégique, plus affectif que rationnel.

Une appartenance à un groupe international représenté dans le monde entier. L'adhésion Best Western International génère des attentes : bénéficier des moyens et de la notoriété de ce groupe.

Ces deux caractéristiques, souhaits d'indépendance, et appartenance à Best Western présentent une contradiction qui développe un certain nombre de conséquences. Elles se manifestent par des expressions repérables dans le guide Best Western France.

Discordance des hôteliers, qui souhaitent que Best Western œuvre pour unir leurs forces, par exemple: regroupement d'hôtels d'une même région, pour mener une politique commerciale commune, mais, qui en même temps, n'arrivent pas à passer à l'acte: ils ne saisissent pas l'occasion de mettre en pratique cette stratégie collective. Nous pouvons citer par exemple, le refus de certains hôteliers de donner leur fichier aux commerciaux de Best Westem France ou encore, l'omission de citer Best Western dans certains de leurs documents de communication, comme des dépliants d'hôtels.

79 Tâche difficile au siège de Best Western qui défend l'idée de politique de groupe, mais, qui offre aux adhérents la possibilité de participer ou de ne pas participer à certaines opérations comme la parution sur le guide séminaire, atomisation des forces, image d'éclatement symbolique.

80 Il y a, dans les termes mêmes utilisés : «Best Western groupe d'hôteliers indépendants », le germe de la contradiction. Comment peut-on être indépendant, et dépendant à la fois, de certaines contraintes du groupe?

Discordance, image d'incohérence et donc de signes externes de manque de cohésion.

Les personnes extérieures au groupe perçoivent mal l'unité, par exemple : omission ou refus réitéré des journalistes de presse, qui, lorsqu'ils parlent en particulier d'un hôtel du groupe Best Western citent peu Best Western dans leurs articles.

La conception du P.C.A ne peut être entamée avant le repérage des indicateurs de cohérence ou de discordance, d'agrégation ou de dissociation. La façon dont les espaces de travail sont représentés et appropriés, ainsi que les signes langagiers, peuvent intervenir dans cette analyse préalable.

Ils manifestent l'ambivalence de leurs significations potentielles, car ils sont susceptibles de s'inverser et de passer, pour le cas présent, de valeurs négatives à des valeurs positives, selon que le système de pilotage est à même de proposer des buts fédérateurs ou non.

L'étude de Best Western France montre que l'hétérogénéité de l'entreprise est un trait constitutif, lié à son histoire, à son activité et à son métier. Le style de management proposé en tient compte au plan symbolique, mais, pris dans les nécessités de gestion, l'occulte au quotidien. 


\section{La structuration de l'espace}

\section{L'environnement de travail : cadre de la personne et miroir de l'organisation}

Fisher $^{5}$ (1991) a montré à la suite de Hall ${ }^{6}$ (1966) et de Goffman ${ }^{7}$ (1973) comment l'espace est un facteur de modèlisation des interactions sociales. L'environnement de travail est un construit dans lequel interviennent la disposition matérielle des locaux et des objets, les marquages de territoires, les modes d'appropriation et les déterminations de sphères personnelles.

Sans reprendre l'ensemble de la problématique développée aujourd'hui par la psychologie de l'environnement de travail, nous utiliserons certaines de ses analyses pour montrer comment les marqueurs d'espace de l'entreprise étudiée peuvent être lus comme des indicateurs de désagrégation. Et ici le terme de désagrégation n'est pas à entendre sous un aspect processuel, mais comme un trait de l'entreprise in nucleo. L'espace que s'est donné l'entreprise Best Western est un reflet de son organisation. En étudiant les différents repères dont disposent les acteurs, nous pensons montrer que le morcellement qui préside au mode de management se retrouve dans l'écologie du travail et prédéfinit un certain partage des temps, des échanges et des actes qui amplifie la structure schizophrénique de l'entreprise. En établissant que le morcellement est une caractéristique définitoire, nous en tirons les conséquences quant au P.C.A : il ne doit pas procéder à une unification de surface, mais travailler à partir de la réalité de l'entreprise.

\section{Un espace de référence éclaté}

La tête du groupe international est à Phoenix, en Arizona. Les dirigeants, en particulier le président, par des voyages assez fréquents, ont orienté une partie de leur espace symbolique de travail vers Phoenix. Sans parler ici de contraintes organisationnelles ou fonctionnelles, nous relèverons simplement comment le point Phoenix, point sans matérialité, existant, sauf pour quelques responsables, surtout par son nom, est utilisé dans le discours pour marquer les effets d'une puissance distante, sinon démiurgique, du moins détentrice d'une information technique ou stratégique :

"A Phoenix, ils ont le manuel du logiciel....", «On va demander à Phoenix si Best Western France peut prendre cette initiative», «Ca va tout à fait dans le sens de Phoenix ».

Le nom de ce lieu est utilisé comme le nom d'un interlocuteur non personnalisé et instantiel. La préférence marquée pour l'option « nom de ville » au détriment d'une autre formulation possible: «Best Western International» ou «les dirigeants du groupe international» montre que ce sont par des lieux, même non connus, simplement nommés, que s'organisent les liens symboliques de l'échange des informations et des décisions.

91 Voilà qui n'a rien d'étonnant pour une entreprise ainsi éclatée en une galaxie de points : le lieu est d'après L. Marin ${ }^{8}$ la stase: la présence immobile qui règle les rapports de coexistence en rendant distincte la place de chaque objet. En distinguant ainsi des lieux, dans une entreprise faiblement matérialisée, on distingue des objets différents, on 
hiérarchise des niveaux de réalité, on planifie l'action grâce à des polarités bien désignées. Dire "Phoenix ", c'est utiliser l'efficience de l'assertif et du directif, c'est classer et ordonner les savoirs sur la succession des tâches à accomplir, c'est utiliser l'instance symbolique de la société mère comme une entité fédératrice.

Ajoutons que Phoenix est la ville où se trouve l'ordinateur central qui régit les réservations Best Western à l'échelle mondiale. L'activité principale de Best Westem, son atout commercial, est la réservation internationale. A tout moment et de n'importe quel terminal Best Western, on peut réserver une chambre dans tout hôtel Best Westem. Or le terme même de réservation exprime bien le caractère clivé de cette activité hôtelière, la gestion d'un énorme stock de chambres. Réserver, c'est promettre qu'une chambre sera occupée, c'est illocutoirement la transformer en bien personnel d'un client pour une, deux ou trois nuits, c'est vendre une durée d'usage, à distance, irréversiblement mais sous réserve. La réservation est donc un acte non conditionnel, du point de vue de l'entreprise, mais aléatoire, du point de vue du client, tributaire de son voyage. Référer une organisation centrale au point Phoenix, c'est peut-être chercher le noyau d'une activité immatérielle, labile, exposée à des interactions brutales : tout en étant autonome, Best Western France a son point focal au loin. Tout en ayant une activité concrète et vitale, vendre des lieux, qui sont des gites, Best Western France n'a jamais de relation empirique à l'aboutissement de son activité. Au fond la notion même de siège, marque de lieu, porte cette ambiguïté. Le siège, c'est là où l'activité de terrain ne se déroule pas, le lieu dépouillé de la matérialité du travail, le lieu de la gestion. Best Western France se structure en deux sièges dupliqués, Paris et Phoenix, qui divisent encore les chances de créer une communauté de travail.

Sans doute le paradoxe de ces demi-autonomies de filiales, toujours emboitées dans une entreprise source, mais engagées à prouver leur maturité au sein du réseau, n'est il pas propre à l'activité hôtelière. L'implantation croissante de grands groupes étrangers sur le territoire national donnera certainement lieu à des études poussées dans ce domaine.

\section{Les hôtels et la topique du nom propre}

Les destinataires de l'activité du bureau parisien sont les hôtels, situés dans toutes les régions françaises. 160 hôteliers qu'on rencontre plus souvent au téléphone qu'en face à face... Ces 160 partenaires constituent un ensemble composite qui eux-mêmes attendent du siège avant tout la commercialisation de leurs nuitées. En fait, l'atout majeur de Best Western, son extraordinaire réseau informatique de réservation mondiale, est source de gains surtout pour les hôteliers des grands centres urbains et touristiques. Les autres risquent à tout moment de se retourner agressivement contre le siège, car l'afflux de clientèle, hors des centres d'affaires et de tourisme, est inférieur aux attentes : «Le bureau, c'est l'exutoire », explique le Président.

Les hôteliers n'ont jamais à l'égard du siège de comportements typifiés, à partir desquels on pourrait élaborer des schémas de résolution de problème. Car, justement, la règle de la S.A. est la mise en relief des différences, et même si le vœu est étouffé sous les nécessités commerciales de standardisation, la relation, elle, - et c'est bien ainsi que l'entendent les hôteliers - est rigoureusement interindividuelle. Alors, comment intérioriser cette galaxie d'hôtels en un univers continu et stable?

96 En fait le seul référent de cet univers hôtelier est le guide Best Western France, dont s'aident longtemps tous ceux qui s'initient à l'entreprise. Sans lui il serait bien difficile 
aux acteurs de se représenter, dans l'interaction, les territoires de leurs partenaires, quand l'expérience visuelle est erratique, liée à un séjour, à une intervention de terrain, à une festivité. C'est donc à partir des entrées du guide que se structure le champ relationnel et fonctionnel: le nom de la région, le nom de la ville, le nom de l'établissement, et enfin le nom du propriétaire ou du directeur. Comme conséquence directe de ce modelage par la cartographie et l'annuaire, le nom de lieu est souvent utilisé pour désigner l'adhérent du groupe :

«Le grand Monarque, c'est Chartres, c'est M. X». «M. Y, le Best Westem d'Andorre », «Mais oui, vous savez, Epinal, c'est lui qui a parlé dans la réunion, c'est M. Z ».

On pressent que cette métonymie est un mode de renforcement d'une «identité topique » appelée à compenser le caractère lacunaire de la représentation de l'entreprise comme un tout.

Pour s'en convaincre, on remarquera que de telles figures sont absentes de la langue de travail ordinaire : dans d'autres univers de travail, même si quelqu'un dit « je vais voir la Direction du personnel, au troisième ", alors qu'il sait bien le nom de la personne qu'il va rencontrer, il y a là l'indication d'un itinéraire réel à parcourir qui dote l'énoncé d'une dimension programmatique. On dit rarement en revanche, «c'est le troisième étage qui a pris la parole en comité de direction»! Ou si on le fait, c'est pour neutraliser volontairement l'identité des individus et insister sur leur (mauvaise) réputation et avec une intention de distance ou de polémique absente de l'emploi que nous signalons ici.

On voit que, dans notre exemple, passer par le nom de lieu est une nécessité qui spatialise la destination de l'activité par des repères symboliques et imaginaires quand les marques concrètes n'en sont pas empiriques.

Le morcellement consubstantiel à l'entreprise a donc produit un renforcement compensatoire des dénominations de lieu.

\section{Chevauchement des espaces et confusion des tâches}

Les territoires existent, mais ne protègent pas l'espace personnel et ne favorisent pas l'appropriation. A partir de ce constat, nous soutenons trois hypothèses :

- l'instabilité des frontières contribue à faire peser sur les relations une revendication permanente, caractérisée par de faibles interactions langagières et des intrusions concrètes ;

- l'absence des frontières matérielles et symboliques est le reflet de l'indéfinition des postes;

- comme la disposition des lieux ne laisse pas de marge, les querelles de territoire sont déportées sur le terrain du travail, les régions et les hôtels ; les interactions conflictuelles ont sedimenté un certain mode de relation brutale avec l'hôtelier, dont celui-ci n'a pas le code, et qui, sous couvert d'un réveil du management, est devenu le style de l'entreprise ;

Le territoire de travail est normalement protégé des intrusions. Ici la structure labyrinthique des bureaux rend les trajets multiples et imprévisibles. À part le grand bureau collectif du service réservation et deux bureaux de la direction, aucun bureau ne bénéficie de la lumière du jour. Mais les cloisons des bureaux sont en verre et subissent un éclairage indirect. Chaque bureau a deux ou trois parois transparentes, qui sont contournées par un couloir. A cause du manque d'aération, les portes sont en outre toujours ouvertes. Chacun à tout moment est dérangé, appelé. L'espace est morcelé. L'espace collectif n'a pas d'existence. Il n'y a pas de centre. Un hall assez vaste est à demiextérieur, puisqu'il est le lieu d'accueil des visiteurs. Une salle de réunion est en sous-sol. 
Seul carrefour des trajets : la salle de photocopie, elle, est à l'entrée des locaux, à la limite de la zone d'accueil et de la zone de travail, traversée par deux ouvertures. Lieu où l'activité, la production des lettres et des notes, se matérialise, se répand et se duplique, elle est le seul endroit de rencontre et d'unité.

Un exemple des transgressions nées de ce plan en boucle: au lieu de faire appeler quelqu'un par un téléphone intérieur ou de lui transmettre une note, on se penche en passant, on "vérifie » qu'il est là, par un regard, un mouvement du corps, et non en engageant une interaction verbale.

Certain de trouver l'interlocuteur, le visiteur entre. Chacun est ainsi imprévisiblement saisi ou dessaisi de ses tâches. Dans de brusques élans, le Directeur peut confier un dossier de poids à une jeune collaboratrice et le lui retirer dans un bruit de blâme. Ces bureaux emmêlés sont-ils le reflet ou la source de ces moments fusionnels brutalement tranchés ?

Ces bureaux fermés mais transparents n'offrent pas d'obstacle au regard. Ils ne favorisent pas non plus des trajets structurés, permettant d'anticiper des rencontres ou de les rechercher. La distribution des bureaux est le reflet du hasard et du turn-over. Expliquée par l'indifférence aux hiérarchies, le travail d'équipe, la nature urgente de toutes les tâches, le côté état de crise, elle ne met pas en cohérence les proximités de bureau et les proximités de fonction.

Bien qu'il y ait territoire, puisque chacun a un bureau, il n'y a pas d'espace protégé du regard et même de l'exploration. L'indéfinition des tâches et le désordre du système d'information font que souvent un document est recherché en l'absence de son détenteur. Ces interactions non régulées deviennent défense et retour agressif.

Un seul service est à l'abri de ce panoptisme, c'est le service comptable. Il officie dans un lieu protégé, templum, sans accès par le couloir, dont le narthex est un bureau sas qui donne lui-même sur le grand bureau de la réservation. On ne sait si ce service est ainsi élevé ou assujetti. Il est l'appareil enregistreur de l'activité, preuve par le chiffre de la réussite ou de l'échec. Le Directeur sauvegarde ses prêtres.

\section{L'exportation des conflits et l'amplification de la dissociation}

109 Goffman (1973) propose trois types de marqueurs de l'appropriation:

- les marqueurs centraux, qui expriment une revendication forte, comme un bureau

- les marqueurs frontières qui séparent des espaces voisins

- les marqueurs signets, qui portent sur la propriété d'un objet.

110 Le tassement des espaces produit chez Best Western un écrasement de ces marqueurs distincts, qui, tout en compromettant l'appropriation, exacerbent a contrario le sentiment de propriété. Ainsi se trouvent en superposition espaces et objets du travail, espaces et objets de la personne.

111 De ces conflits de personne, le Président est protégé, car il n'est qu'un hôte sporadique du bureau du directeur. Ils altèrent cependant la conduite des affaires en particulier entre le Directeur et la Directrice de la Communication. Leur conflit s'exporte hors de ce lieu étroit, en direction des hôteliers, qui font les frais, sans avoir les clés des messages, de demandes contradictoires ou redoublées, de politiques divergentes, d'initiatives annoncées et brusquement enterrées. A distance et sans possibilité de correctif, cette 
situation a des effets dévastateurs sur la crédibilité du siège. Mais les acteurs, englués dans leurs soucis, n'ont pas conscience de gérer ainsi une entreprise à la personnalité malade.

Lieu «boule ", il oblige à la fusion, quand tout signe le morcellement. N'est-il pas le symptôme de la contradiction entre les énoncés littéraux: unis, ensemble, une seule identité, un groupe et le sens conflictuel qui se dégage des messages internes du groupe ?

A partir d'un tel bilan, on peut dégager des lignes d'intervention: l'attribution des bureaux peut être rediscutée de façon à faire de la distribution des espaces la projection des relations fonctionnelles et organisationnelles. Penser des règles de circulation et de contact, c'est pour les acteurs un vecteur de parole qui met au jour l'histoire du siège et les effets des styles de management, sans pour autant mettre au pilori un « responsable».

\section{La communication écrite interne et externe}

\section{Les marqueurs graphiques et linguistiques de la dissociation}

114 Un fax hebdomadaire, émanant du siège à destination des 160 hôtels, donne les informations fonctionnelles ou relationnelles qui expriment la vie du groupe. Ce fax est coordonné par la directrice de la communication mais est composé à partir des écrits des différents acteurs du siège.

Quelques exemples suffiront à montrer la difficulté à faire coexister des mondes différents et à faire écrire ensemble des individus aux points de vue hétérogènes.

Les titres représentent des strates différentes, jamais régularisées, de l'entreprise. Serviront de titres le nom d'un service (Achats, Communication), une décision de fonctionnement (Réunion de région), une modalité appréciative (Métamorphose), une injonction (Attention!), une entité du groupe (Best Western Australia).

117 Ce nivellement montre que la parole commune repose sur des agglomérats accidentels, qui mêlent assertion et injonction sans hiérarchie, exprimant de façon confuse des catégories de l'action, de l'organisation, du jugement. L'hétérogénéité des textes pourrait se justifier si une organisation graphique lui donnait corps. Or, malgré les possibilités offertes par la P.A.O, les pavés restent nucléaires, sans possibilité d'une lecture en réseau ou par sélection de thèmes.

118 Les marques de renonciation présentent la même anarchie: un pavé titré " communication » propose le message suivant :

119 « COMMUNICATION

120 Je remercie les "spécialistes" de bien vouloir prendre contact avec MM. qui aurait besoin de tuyaux pour organiser des journées de chasse.

Réunions: pour nous permettre de travailler au mieux, pensez à vous munir des documents demandés (notre courrier du 23 juillet)

122 Les chefs de service se feront un plaisir de vous recevoir le vendredi matin, merci de prendre rendez-vous».

Dans le même pavé, trois énonciateurs différents : «je », (sans signataire) « nous » (sans définition de l'ensemble), «les chefs de service » (retour à l'organisation), sans que le contexte permette une justification claire de ces glissements. Dans la première demande, «je» se met en position de relais entre un hôtelier et tous les autres. L'initiative 
personnelle de l'interaction provoque le «je». Dans la phrase suivante, le "nous» pourrait être autant la puissance invitante, du reste non nommée et non délimitée, que les participants. Dans la troisième phrase, «les chefs de service » semblent utiliser le «plaisir » comme antiphrase de la coercition. Et l'accueil ainsi proposé semble tout de même considérer le visiteur comme nettement indésirable !

Les exemples de phrases séductrices renversant un reproche sont légion: "les formulaires d'adhésion et les tracking forms sont précieux : ils coûtent très cher. Merci de ne pas les égarer.»

$\mathrm{Au}$ point qu'on peut se demander si le service communication est une instance de mise en relation des adhérents ou de gestion de la menace. C'est un trait dominant de toute la communication interne. Les injonctions, le message punitif écrasent les appels à la convivialité, les marques de gratitude et les encouragements. Non tant qu'il s'agisse d'un paternalisme autoritaire. La communication interne se pose plutôt comme un discours d'experts : celui de la lucidité du siège qui maîtrise l'information sur l'environnement. La gravité de la situation provoque la précipitation tragique... Agir vite, parler cru, dépouiller l'innocence des hôteliers protégés des loups par le siège...De notre point de vue d'observateurs, ce sont les symptômes de l'anxiété d'un management conflictuel, qui surtout, n'a pas su lire à temps certains signes du changement des positions des hôteliers, passés progressivement d'une lecture très restrictive de la réalité (" mon établissement », " ma clientèle », « mon chiffre d'affaires ») à un discours plus stratégique.

\section{Récente cohésion des adhérents, décomposition du siège}

La communication externe a construit une image cohérente d'un groupe d'hôteliers indépendants, reposant sur des valeurs communes, la qualité et le service. Cette image occupe le guide, les plaquettes de présentation de l'entreprise, les communiqués de presse. Et si le mot groupe, utilisé pour la désignation externe, évoque un ensemble d'éléments réunis en un tout, ouverts aux flux externes, modifié en permanence, mais maintenant sa cohésion dans la durée, on remarque que c'est le mot chaîne qui représente Best Westem en communication interne. Avec une tout autre connotation, celle de la succession de maillons, à parcourir un par un, qu'on peut augmenter mais pas modifier sans rupture. On trouve également notre label Best Western : et c'est tout le parler-vrai commercial, comme si le discours d'une symbolique des valeurs était bon pour le service communication, comme l'attrape-nigaud à la mode. Mais avec les adhérents, on tombe le masque. Et on tient un discours de prescription, d'autorité, d'urgence, de menace. Le courrier contentieux parle tout de suite d'exclusion. Les procédures sont engagées au lance-pierre, sans annonce, sans explication, entre hommes d'affaires. Au siège, le directeur compte les fautes et fait tomber les têtes. En un an d'observation, quatre chefs de service ont été licenciés.

Or ce que la Directrice de communication avait engagé avec le Président, et, en apparence avec le Directeur, est en relative cohésion avec les vœux des hôteliers. Dans leur territoire nucléaire, leur sentiment d'unité s'est éveillé, à l'insu du Directeur. La participation aux réunions de région augmente, l'intérêt pour la conception de la campagne de notoriété ne se dément pas. Les liens qui se tissent, le sentiment partagé de défendre ensemble l'hôtellerie indépendante créent des attentes à l'égard du bureau. Attentes déchiffrées superficiellement comme les nouveaux impératifs d'adhérents toujours plus exigeants, 
parasitant le travail de fond. Et c'est finalement ce récent référent commun, non reconnu au siège, qui sera un des vecteurs d'expression de la crise.

Au siège, l'espace illisible est doublé par un temps qui s'accélère : toujours plus urgente, la communication écrite avec les hôteliers ne prévoit même pas de délai de réponse. Les responsabilités sont épisodiques, l'équipe des chefs de service se renouvelle de $60 \%$ en un an, les licenciements et les départs touchent surtout le personnel de Direction. Le Directeur valorise les ascensions fulgurantes de jeunes secrétaires, obligées d'assumer le travail d'un service sans tête. Les adhérents, eux, s'attendaient à la fin de ces pratiques. On leur parle stabilité, union, stratégie sur le long terme. Or leurs interlocuteurs changent et le départ de la Directrice de communication laisse s'accroître l'écart entre les discours du Président et ceux du Directeur.

\section{Une entreprise morcelée en mutation}

morcellement que nous avons présenté comme constitutif de l'entreprise, par son histoire, son activité et son organisation, est accéléré par les conflits de personnes que la désagrégation territoriale exporte dans chaque hôtel du groupe.

Il n'est pas en soi pathogène: l'exubérance de l'entreprise en réseau est une qualité contraignante, qui impose une mutabilité permanente et donc un management souple et attentif.

131 Le siège, refermé sur ses angoisses et bloqué par sa position de relais d'une entreprise mondiale, a au fond voulu soigner les signes de la désagrégation, comme s'ils le menaçaient lui-même, par une chirurgie du champ de bataille, sans reconnaître leur nature essentielle.

Peut-être faudrait-il entendre la leçon médiévale, lue par M. Bakthine dans l'œuvre de Rabelais. Dans le corps plein de saillies et de centres que dessine la culture du Moyen Age, par sa littérature et sa sculpture, se devinent le changement permanent, la création utopique et la conscience cosmique :

"Le corps grotesque est corps en mouvement. Il n'est jamais prêt ni achevé: il est toujours en état de construction, de création et lui-même construit un autre corps: de plus ce corps absorbe le monde et il est absorbé par ce dernier (...) Dans la chaîne infinie de la trie corporelle, les images grotesques fixent les parties où un maillon est engagé dans le suivant, où la vie d'un corps naît de la mort d'un autre, plus vieux. $"^{9}$

Cet idéal de renouvellement continu est progressivement inhibé par la culture officielle : «Le trait caractéristique du nouveau canon est un corps parfaitement prêt, achevé, rigoureusement délimité, montré de l'extérieur, non mêlé, individuel et expressif ${ }^{10}$.

A la polysémie du corps grotesque succède le corps moderne refermé, qui parle de sa seule voix, efface les saillies et se clôt dans une identité statique.

Une entreprise telle que Best Western a la chance de porter en elle, par sa structure en réseau, les germes d'une évolution pleine de sens. Le management, tout en concevant les différences comme une qualité intrinsèque du groupe, parvient mal à s'ajuster au jour le jour aux exigences concrètes qui en découlent.

137 Comment sous une seule bannière communiquer à l'extérieur une image unie de cette structuration éclatée en cascade? 
138 Dans cet univers à la symbolique émiettée, ou au moins floue, foyer d'anxiété, l'élaboration de valeurs fortes communes semble incontournable.

\section{Le Plan Commun d'Action, un objectif suspendu}

139 Notre recommandation de départ préconisait l'élaboration du Plan Commun à partir de l'accord de trois acteurs : le directeur, le président directeur général et la directrice de la communication. Ensemble ils ont su définir des objectifs de politique générale :

- le doublement en trois ans du nombre des adhérents ;

- le développement de la qualité des services des hôtels ;

- la stabilisation d'un personnel de qualité, en renforçant son association à l'entreprise et en lui accordant une meilleure considération ;

- l'uniformisation des taux de remplissage des établissements à $70 \%$.

Pour atteindre ces objectifs, il fallait d'emblée cultiver les signes d'une convergence des buts et d'un partage des valeurs. Les actions de communication s'y sont employées, en engageant une réflexion sur la qualité Best Western France et ses preuves, en définissant les critères et les procédures de recrutement des nouveaux hôteliers. Pour dynamiser la créativité et l'exigence de qualité, les hôteliers ont choisi de passer par un contrôle de qualité, seul moyen de rendre comparables des établissements par nature singuliers. Ils ont en outre travaillé ensemble à créer un concept petit déjeuner; l'analyse des questionnaires clients avait en effet montré l'amplification de l'opinion du client selon son jugement sur le petit déjeuner.

Avec un personnel à rotation rapide et souvent saisonnier, la gestion des ressources humaines est encore à l'état endémique, en particulier dans les établissements de taille modeste. Les hôteliers y viennent, par le biais de stages destinés au personnel d'accueil et d'encadrement qui sont un moyen de diffuser et de s'approprier un langage et des représentations communes.

Ces tâches partagées ont été une avancée vers la formulation de valeurs identifiées. Ainsi, en cherchant lors de réunions de région les moyens graphiques de présenter les établissements dans leur originalité mais aussi dans la généralité de l'appartenance à la chaîne, les hôteliers ont pu améliorer leur représentation des caractéristiques de leur établissement et passer d'un questionnement de type commercial à une interrogation sur la valeur symbolique de l'attachement.

Ils ont alors délégué à une commission la responsabilité de préparer le cahier des charges de la campagne de notoriété. Pour sélectionner un projet, pour en négocier la stratégie, pour enclencher les actions locales d'accompagnement de la campagne, ils n'ont plus renoncé à ce double effort : cohérence de relations avec leurs partenaires, cohésion d'une approche commune.

Des changements concrets en portent la trace : au siège, travail sur l'homogénéisation des messages écrits, à partir d'une réflexion sur les attentes et les points de vue des destinataires, quant à l'information et quant à la communication; pour toute la société, établissement d'une charte de communication, reposant sur des règles unifiées de rédaction, de mise en page, de code couleurs; pour les établissements, extériorisation de l'appartenance par le renforcement d'une signalétique. 


\section{NOTES}

1. Lacan, J, 1966, Écrits, Le Seuil page 104.

2. Cabay, M., Magnétoscope et formation à l'observation des attitude corporelles. Contribution à une étude du langage du corps. Thèse 1978 Toulouse.

3. Montagner, H., L'Enfant et la communication - Paris - Éditions PERNOUD-STOCK.

4. Watlzawick P., Helmick Beavin J., Jackson Don. D. 1979, Logique de la communication, traduction française, J. Morche, Paris, Le Seuil.

5. Fischer G, 1991, «Espace, identité et organisation », in Chanlat, J-F., (sous la direction de) L'individu dans l'organisation, Québec, Presses de l'Université Laval et éditions Eska.

6. Hall, E.-T.1966, La dimension cachée, Paris, Éd. Seuil.

7. Goffman. E., 1973, La mise on scène da la vie quotidienne, 2 vol. Paris, Éditions de Minuit.

8. Marin, L., 1992. Lectures traversières. Paris, Albin Michel.

9. Bakhtine. M-, 1929, L'œuvre de François Rabelais et la culture populaire au Moyen Age, 1979 pour la traduction française, Gallimard, pp. 315 et 316.

10. Bakhtine, $M, 1929$, op. cit. p. 318. 


\section{RÉSUMÉS}

Le Plan Commun d'Action (P.C.A), élaboré par les forces vives de l'entreprise, est la condition de la communication globale. Notre étude montre a contrario que les signes de morcellement dans une société ne sont pas un obstacle à l'unification à condition d'être analysés comme des traits constitutifs. L'histoire de l'entreprise, les projets des acteurs et leurs interactions laissent un sédiment qui modèle l'organisation et construisent le sens des valeurs nécessaires à la maturation du projet de communication globale. Une condition est cependant nécessaire à la mise en œuvre d'un Plan commun d'action : l'unité du directoire, sans laquelle le développement est limité à la mise au Jour de signes partagés.

\section{AUTEURS}

\section{MICHÈLE GABAY}

Michèle Gabay est professeur d'université à l'IUFM de Créteil et à HEC, docteur ès lettres, section information et communication.

\section{SOPHIE PÈNE}

Sophie Pène est attachée d'enseignement et de recherche à l'IUT de Paris, Université René Descartes-Paris V. 